\title{
Severe bone marrow suppression following single-dose methotrexate treatment for ectopic pregnancy
}

\author{
Betül Dündar1, Burcu Dinçgez Çakmak¹, Gülten Özgen¹, Fatma Ketenci Gencer², Burcu Aydın Boyama ${ }^{3}$ \\ ${ }^{I}$ Department of Obstetrics and Gynecology, University of Health Sciences, Bursa Yüksek Ihtisas Training and Research Hospital, \\ Bursa, Turkey \\ ${ }^{2}$ Department of Obstetrics and Gynecology, University of Health Sciences, Gaziosmanpaşa Taksim Training and Research Hospi- \\ tal, İstanbul, Turkey \\ ${ }^{3}$ Department of Obstetrics and Gynecology, Medipol University, Esenler Hospital, İstanbul, Turkey
}

\begin{abstract}
Methotrexate is associated with multisystem side effects which depend on the dosage, route of administration and length of treatment. Bone marrow suppression is a very rare but one of the most mortal complications. A case of severe bone marrow suppression following single dose methotrexate for ectopic pregnancy was presented in this study. A 33-year-old woman, diagnosed as ectopic pregnancy, was given single-dose intramuscular methotrexate of $50 \mathrm{mg} / \mathrm{m}^{2}$. On 10th day patient was admitted with lethargy, irritability, vomiting, hematemesis, diarrhea, oral mucositis and skin rash. Laboratory findings revealed severe bone marrow suppression. Supportive treatment together with antibacterial/ antifungal drugs and granulocyte colony stimulating factors were administered. On the 3rd day of treatment, mucositis and oral intake began to improve. After 1 week complete blood count was normal. Methotrexate may have mortal clinical consequences even with very low doses. Therefore, a careful patient history for patient selection and closer clinical follow-up are essential.
\end{abstract}

Eur Res J 2017;3(3):285-289

Keywords: Bone marrow suppression; ectopic pregnancy, methotrexate, side effects, single-dose regimen

\section{Introduction}

Ectopic pregnancy is defined as implantation of blastocyst somewhere other than uterine cavity with an incidence of $1-2 \%$ [1]. The treatment modalities of ectopic pregnancy vary from expectant management to radical surgery. Methotrexate (MTX) is the most commonly used agent in medical treatment of ectopic pregnancy [2].
MTX, which is an antifolate cytotoxic drug inhibiting cellular proliferation, can be used as a chemotherapeutic or immunosuppressive agent in several conditions such as leukemia, lymphomas, lung, breast and bladder cancer, multiple sclerosis, rheumatoid arthritis, Crohn's disease. In daily gynecologic practice, it is most commonly used in 
treatment of ectopic pregnancy and gestational trophoblastic neoplasia [3]. Although the indication of MTX treatment has a broad spectrum, it has several side effects some of which are mortal.

During MTX treatment, mucositis which is generally associated with gastrointestinal symptoms such as nausea and vomiting, and hematological side effects are quite common. In addition to these, pneumonia, pleural effusion, pericarditis and pericardial effusion arising from serositis could be encountered during treatment. However, bone marrow suppression is a very rare but one of the most mortal complications [3, 4]. The side effects of MTX depend on the dosage, route of administration and length of treatment period.

In this study, we presented a case of severe bone marrow suppression following single dose of MTX treatment for ectopic pregnancy.

\section{Case Presentation}

A 33-year- old, gravida 2 parity 2 women with a history of cesarean section and with no other medical or gynecologic history was admitted to emergency room with secondary amenorrhea and vaginal bleeding. In general physical examination, there was minimal tenderness on right adnexal area. In gynecologic assessment, there was minimal vaginal bleeding and painful cervical movements. In transvaginal ultrasonography, endometrial thickness was $8 \mathrm{~mm}$ and there was $15 \times 13 \mathrm{~mm}$ hyperechogenic mass not including fetal nod which is coherent with ectopic pregnancy at right adnexal area. Serum betaHCG level was 1,374 mIU/ml. During clinical follow up, the serum beta-HCG value at $48^{\text {th }}$ hour was 1,532 $\mathrm{mIU} / \mathrm{ml}$. In detailed laboratory assessment it was found that white blood count (WBC): 4,200/ $\mathrm{mm}^{3}$, neutrophil: $\left.2,300 \times 1,000 / \mathrm{mm}^{3}\right)$, platelet (PLT): 295,000/mm 3 , hemoglobin (Hb): $11.4 \mathrm{~g} / \mathrm{dl}$, hematocrit (HCT): 33.7 (\%), MCV: $92.2 \mathrm{fL}$, vitamin $\mathrm{B}_{12}: 327$ $\mathrm{pg} / \mathrm{mL}$, folic acid: 21 , alanine aminotransferase: 18 IU/L, aspartate aminotransferase: $16 \mathrm{IU} / \mathrm{L}$, creatinine: $0.8 \mathrm{mg} / \mathrm{dL}$, urea: $17 \mathrm{mg} / \mathrm{dl}$, INR: 1.3 and APTT: 36 min. No pathological finding was observed in chest Xray. She was hospitalized with the diagnosis of ectopic pregnancy. Since it is not recommended to use antibiotics in patients without any risk factors, we did not give any antibiotics. The patient, who did not have any contraindications for MTX treatment, was given single-dose intramuscular MTX of $50 \mathrm{mg} / \mathrm{m}^{2}$ for ectopic pregnancy. After 24 hours following MTX dose she developed severe nausea and vomiting unresponsive to metoclopramide and ranitidine hydrochloride.

On the $1^{\text {st }}, 4^{\text {th }}$ and $7^{\text {th }}$ days of MTX treatment, betaHCG was 1,560, 1,645 and 1,905 $\mathrm{mIU} / \mathrm{ml}$, respectively. Because of increasing serum level of beta-HCG, a second single dose of MTX was planned but because of patient's severe nausea and vomiting unresponsive to medical treatment and development of acute abdomen without any clinical and/or sonographic findings of acute hemorrhage, diagnostic laparoscopy was performed. In abdominal exploration, a $20 \times 10 \mathrm{~mm}$ ectopic mass was observed in the ampulla of right uterine tube and right salpingectomy was performed. On the 1st postoperative day, patient was discharged without any early complications. After two days, patient was admitted to hospital with fever, severe vomiting accompanied by hematemesis, diarrhea, lethargy, irritability and confusion. Patient's vital findings were as follows: arterial blood pressure was $100 / 70 \mathrm{mmHg}$, heart rate was 80 beats per minute and body temperature was $38^{\circ} \mathrm{C}$. In physical examination the skin was dry. There were multiple eruptions particularly located on the trunk and pigmentation of face, subconjunctival hemorrhage and diffuse oral mucositis disturbing oral intake. She was suffering from oliguria and hematuria. The reason of hematemesis and diarrhea are explained by diffuse mucositis affecting gastrointestinal tract including oral mucosa, which consequently results in dehydration. As the severity of diffuse mucositis increases dehydration worsens gradually and at the end symptoms like lethargy, irritability and confusion arise. Mucositis is not only associated with gastrointestinal tract. Also, urinary tract mucosa is affected in case of MTX toxicity. Together with mucositis affecting urinary tract severe dehydration are most possible underlying reasons for hematuria. Respiratory and cardiovascular system examination did not reveal any pathological findings. Abdominal examination revealed diffuse abdominal tenderness accompanied by hyperactive bowel sounds.

In laboratory assessment it was found that WBC: $1,200 / \mathrm{mm}^{3}$, neutrophil: $300 \times 1,000 / \mathrm{mm}^{3}$, PLT: 200,000/ $\mathrm{mm}^{3}, \mathrm{Hb}: 10.3 \mathrm{~g} / \mathrm{dl}, \mathrm{HCT}: 31 \%$, alanine aminotransferase: $\quad 17 \quad$ IU/L, aspartate aminotransferase: $13 \mathrm{IU} / \mathrm{L}$, creatinine: $0.9 \mathrm{mg} / \mathrm{dL}$, urea: $11 \mathrm{mg} / \mathrm{dl}$, INR: 1.1 and APTT: $32 \mathrm{~min}$, CRP: 10 $\mathrm{mg} / \mathrm{L}$. No pathological finding was observed in electrocardiography and chest x-ray. She was 
hospitalized with the diagnosis of suspected bone marrow suppression.

Since, the patient's oral intake was disturbed intravenous fluid, total parenteral nutrition was given as supportive treatment. Urine and gaita samples were collected for direct examination. After collecting samples for cultures, prophylactic antibiotherapy protocol composed of ciprofloxacin $400 \mathrm{mg} ; 2 \mathrm{x} 1$, amoxicillin/clavulanic acid $1 \mathrm{gr} ; 2 \mathrm{x} 1$, fluconazole 150 mg; $1 \mathrm{x} 1$ were started. As palliative treatment folinic acid $50 \mathrm{mg}$; 1x1, antifungal and analgesic containing oral suspensions were added. Direct examination of urine and gaita and cultures did not reveal any pathological findings. For neutropenia, filgrastim (recombinant methionyl human granulocyte-colony stimulating factor) $300 \mathrm{mcg}$; $1 \mathrm{x} 1$ was given for 4 days. The complete blood count and CRP values of the patient during filgrastim treatment were given in Table 1. As the patient's monocyte count tended to increase patient's mucositis clinic was began to resolve and patient's oral intake returned to normal.

The reason for using monocyte count in clinical follow up is that our patient was mainly suffering from diffuse mucositis affecting all gastrointestinal tract including oral mucosa and monocyte count closely correlates with the recovery of oral lesions. Therefore, we mainly followed monocyte count to determine clinical recovery of most of the symtoms which were associated with oral intake and dehydration.

The patient was discharged from hospital and after one week following hospital discharge, laboratory findings of patient were completely normal.

\section{Discussion}

The treatment modalities currently being used in clinical management of ectopic pregnancy are composed of expectant management, medical treatment and surgical approach. Today in medical treatment of ectopic pregnancy, considering its cost effectiveness and opportunity of fertility preservation, MTX has a well-proven role if patient is selected appropriately.

MTX can be administered as single-dose, doubledose or multi-dose therapy. Stovall et al. [5] were the first researchers to explore the consequences of MTX therapy in treatment of ectopic pregnancy and they reported a cure rate of $96.7 \%$ with single-dose regimen. Their single-dose therapy and monitoring protocol is being currently used worldwide by clinicians. Following administration of a single-dose intramuscular MTX of $50 \mathrm{mg} / \mathrm{m}^{2}$, serum beta-HCG levels are monitored by measurement of its blood levels on the $1^{\text {st }}, 4^{\text {th }}$ and $7^{\text {th }}$ days and weekly until complete recovery. Determination of $\geq 15 \%$ of decrement in serum beta-HCG levels between $4^{\text {th }}$ and $7^{\text {th }}$ days of MTX administration is accepted as successful with a positive predictive value of $93 \%$, a sensitivity of $93 \%$ and a specificity of $84.2 \%$ [6]. If the expected decline does not occur, another MTX dose of $50 \mathrm{mg} / \mathrm{m}^{2}$ can be given intramuscularly [7]. It was reported that nearly $20 \%$ of patients require additional MTX dose(s) to reach the expected serum beta-HCG levels.

However, to have successful results with MTX treatment appropriate patient selection is essential. Treatment failure is not uncommon if the upper limit of pretreatment serum beta-HCG level exceeds 3,000$5,000 \mathrm{IU} / \mathrm{L}$ and/or if there is a gestational sac larger than 3-4 cm in size and/or a fetal node with cardiac activity $[3,7]$.

In our case the patient who did not have any contraindications for MTX therapy with a pretreatment serum beta-HCG level of 1,532 IU/L was given a single-dose intramuscular MTX of $50 \mathrm{mg} / \mathrm{m}^{2}$.

Table 1. Complete blood count and CRP values during filgrastim treatment.

\begin{tabular}{lccccccc}
\hline $\begin{array}{c}\text { Day of } \\
\text { treatment }\end{array}$ & $\begin{array}{c}\mathrm{Hb} \\
(\mathrm{g} / \mathrm{dl})\end{array}$ & $\begin{array}{c}\text { MCV } \\
(\mathrm{fL})\end{array}$ & $\begin{array}{c}\text { Monocyte } \\
\left(/ \mathrm{mm}^{3}\right)\end{array}$ & $\begin{array}{c}\mathrm{NEU} \\
\left(\mathrm{x} 1000 / \mathrm{mm}^{3}\right)\end{array}$ & $\begin{array}{c}\text { WBC } \\
\left(/ \mathrm{mm}^{3}\right)\end{array}$ & $\begin{array}{c}\text { PLT } \\
\left(/ \mathrm{mm}^{3}\right)\end{array}$ & $\begin{array}{c}\text { CRP } \\
(\mathrm{mg} / \mathrm{L})\end{array}$ \\
\hline $1^{\text {st }}$ & 9.8 & 87.4 & 0.21 & 700 & 1,600 & 100,000 & 14 \\
$2^{\text {nd }}$ & 9.3 & 86.5 & 0.18 & 500 & 1,700 & 70,000 & 15 \\
$3^{\text {rd }}$ & 8.3 & 82.4 & 0.25 & 900 & 1,830 & 50,000 & 14 \\
$4^{\text {th }}$ & 8.1 & 88.4 & 0.37 & 1,100 & 2,100 & 180,000 & 17 \\
\hline
\end{tabular}

$\mathrm{Hb}=$ hemoglobin, $\mathrm{MCV}=$ mean corpuscular volume, $\mathrm{WBC}=$ white blood count, $\mathrm{NEU}=$ neutrophil, $\mathrm{PLT}=$ platelet, $\mathrm{CRP}=\mathrm{C}$-reactive protein 
Serum beta-HCG level was monitored on the $1^{\text {st }}, 4^{\text {th }}$ and $7^{\text {th }}$ days of MTX administration. Serial serum beta-HCG measurements revealed an increase whereas it was expected to decrease. Because of increasing serum beta-HCG levels a second-dose MTX was planned. But since the patient had severe nausea and vomiting unresponsive to treatment and developed acute abdomen, laparoscopic exploration was preferred instead of giving a second-dose MTX.

MTX, which competes with folinic acid functioning in DNA synthesis and cell proliferation, prevents division of cytotrophoblasts resulting in regression of ectopic pregnancy tissue [8]. Although, life-threatening complications of MTX treatment are not very common, because of its non-specific effects on cell division, MTX toxicity may cause severe clinical consequences. Common side effects were reported to be observed in less than $10 \%$ of cases [9]. MTX particularly affects rapidly dividing cells of hematological, gastrointestinal and dermatological systems. As a consequence, most frequently reported signs and symptoms are neutropenia, generalized myelosuppression, nausea, vomiting, diarrhea, gastrointestinal inflammation, generalized erythema, rash, photosensitivity and alopecia [3]. Although, it was reported that side effects were dose-dependent and increased as the duration of treatment increased, in the present case the side effects were developed following only a single-dose MTX of $50 \mathrm{mg} / \mathrm{m}^{2}$. She suffered from generalized myelosuppression, ulcerative stomatitis, gastrointestinal inflammation and generalized erythema.

The underlying mechanism of toxicity may be methylenetetrahydrofolate reductase (MTHFR) 2 mutations resulting in hypersensitivity of MTX, gene polymorphism in the folate metabolic pathway, decreased renal excretion due to any reason such as dehydration, ingestion of cola containing drinks and coincidental use of some drugs [9-11].

After our patient developed the side effects associated with MTX her family members gave us the information that there were people in their village who had once used MTX for several reasons such as malignancies and rheumatologic diseases and developed mortality and morbidity because of associated complications following treatment. For that reason, in the present case we thought that the probable cause of toxicity may have a genetic basis.

In this case, we gave folinic acid and neupogen together with antibiotic and antifungal agents. Our patient relieved clinical signs and symptoms of toxicity on the $4^{\text {th }}$ day of treatment and she became normal as her oral intake increased.

In the literature, we came across only one patient who developed life-threatening neutropenia following single-dose MTX therapy for ectopic pregnancy [12]. In spite of the fact that side effects were reported to be as rare as $2 \%$, its consequences may be lifethreatening and even may occur with low doses of MTX for non-malignant conditions [3]. Strategies, like measuring blood levels of MTX during treatment, considering plasma kinetics of MTX may be helpful in preventing its toxicity and improving safety [13].

\section{Conclusion}

In conclusion, since MTX metabolism is prone to be affected by several conditions and its blood levels can vary because of any intervening factors, a careful patient history for a better patient selection and closer clinical follow-up are essential. It should be better to keep in mind that although, its side effects are quite rare they may have mortal clinical consequences even with very low doses given for treatment of benign conditions.

\section{Informed consent}

Written informed consent was obtained from the patient for the publication of this case report.

\section{Ethical approval}

All procedures performed in studies involving human participants were in accordance with the ethical standards of the institutional and/or national research committee and with the 1964 Helsinki declaration and its later amendments or comparable ethical standards.

\section{Conflict of interest}

The authors declared that there are no potential conflicts of interest with respect to the research, authorship, and/or publication of this article.

\section{References}

[1] Boufous S, Quartararo M, Mohsin M, Parker J. Trends in the incidence of ectopic pregnancy in New South Wales between 1990-1998. Aust N Z J Obstet Gynaecol 2001;41:436-8.

[2] Uğurlucan FG, İyibozkurt AC, Çetin C, Nehir A, Akhan S. [Methotrexate treatment for ectopic pregnancy: Factors affecting treatment outcome]. Ege Tip Dergisi 2013;52:199-204. [Article in Turkish)

[3] Skubisz MM, Tong S. The evolution of methotrexate as a 
treatment for ectopic pregnancy and gestational trophoblastic neoplasia: a review. ISRN Obstet Gynecol 2012;2012:637094. [4] Dündar B, Karalök A, Üreyen I, Gündoğdu B, Öçalan R, Turan T, et al. Case report: Methotrexate-induced pericardial effusion. J Turk Ger Gynecol Assoc 2013;14:48-9.

[5] Stovall TG, Ling FW, Gray LA. Single-dose methotrexate for treatment of ectopic pregnancy. Obstet Gynecol 1991;77:754-7. [6] Kirk E, Condous G, Van Calster B, Haider Z, Van Huffel S, Timmerman D, et al. A validation of the most commonly used protocol to predict the success of single-dose methotrexate in the treatment of ectopic pregnancy. Human Reprod 2007;22:858-63. [7] Soliman KB, Saleh NM, Omran AA. Safety and efficacy of systemic methotrexate in the treatment of unruptured tubal pregnancy. Saudi Med J 2006;27:1005-10.

[8] Sivalingam VN, Duncan WC, Kirk E, Shephard LA, Horne AW. Diagnosis and management of ectopic pregnancy. J Fam Plann Reprod Health Care 2011;37:231-40.

[9] Dasari P, Sagili H. Life-threatening complications following multidose methotrexate for medical management of ectopic pregnancy. BMJ Case Rep 2012;2012:bcr0320126023.

[10] Faganel Kotnik B, Grabnar I, Bohanec Grabar P, Dolzan V, Jazbec J. Association of genetic polymorphism in the folate metabolic pathway with methotrexate pharmacokinetics and toxicity in childhood acute lymphoblastic leukaemia and malignant lymphoma. Eur J Clin Pharmacol 2011;67:993-1006.

[11] Santucci R, Levêque D, Herbrecht R. Cola beverages and delayed elimination of methotrexate. $\mathrm{Br} \mathrm{J}$ Clin Pharmacol 2010;70:762-4.

[12] Isaacs JD, McGehee RP, Cowan BD. Life-threatening neutropenia following methotrexate treatment of ectopic pregnancy: a report of two cases. Obstet Gynecol 1996;88:6946.

[13] Stoller RG, Hande KR, Jacobs SA, Rosenberg SA, Chabner BA. Use of plasma kinetics to predict and prevent methotrexate toxicity. N Eng J Med 1977;297:630-4. 